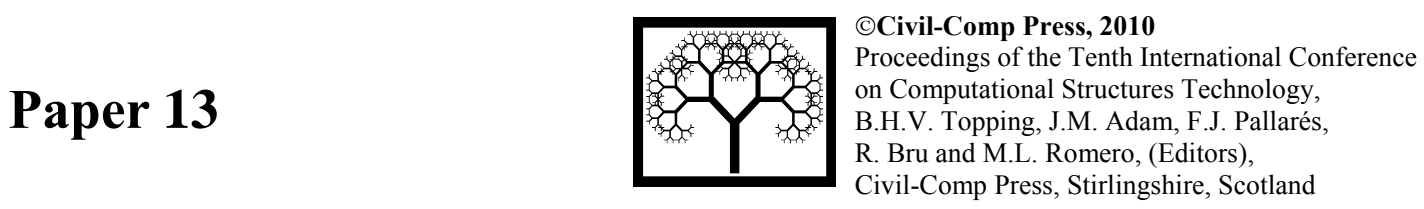

\title{
The Impact of Structural Deformations of Wheelset and Rail on Wheel-Rail Contact
}

\author{
I. Kaiser \\ Institute of Robotics and Mechatronics \\ German Aerospace Center (DLR), Wessling, Germany
}

\begin{abstract}
The distribution of the stresses in the rolling contact of a railway vehicle can be very sensitive to changes of the relative kinematics. Such changes can be caused by displacements of the wheelset in the track, but also by structural deformations of the wheelsets and the rails. A detailed knowledge of the stress distribution in the wheelrail contact is the basis for an accurate analysis of the wear. Therefore, a simulation model is developed, which describes a passenger coach running on a track. In this model, the structural dynamics of the wheelsets and rails are taken into account, and a detailed model for the rolling contact is used. Using this model, the influence of the structural dynamics of wheelsets and rails on the wheel-rail contact is investigated.
\end{abstract}

Keywords: Railway vehicle, vehicle-track interaction, wheel-rail contact, non-elliptic contact, elastic wheelset, elastic rail, elastic multibody system.

\section{Introduction}

The modelling of a railway vehicle as a multi-body system (MBS) consisting of rigid bodies covers several problems, e.e. the low-frequent behaviour $(f<20 \mathrm{~Hz})$ of the vehicle which is related to the topics of running safety and riding comfort. But for several other problems like noise and wear, this modelling reaches its validity limits.

Especially the description of the wear requires a more detailed modelling of the wheel-rail contact. It is self-evident that wear can only occur where wheel and rail are actually in contact. Furthermore, the contact area consists of an area of adhesion and an area of sliding. For a sufficient modelling of the wear, an accurate determination of these zones is necessary. In contrast, for the calculation of the vehicle's motion only the resulting forces acting in the wheel-rail contact are required, so that local errors of the stress distribution have no strong impact. 
Of course, a more detailed modelling of the contact only makes sense, if it is integrated into a refined model of the vehicle-track system. In such a refined model, the structural dynamics of the wheelsets and the rails are taken into account, because deformations of the wheelsets and the rails cause changes in the relative kinematics of the wheel rim and the railhead. Of course, these deformations are small compared to the motions of the entire wheelset, but some combinations of wheel and rail profiles can be very sensitive even to small changes in the kinematics.

In many cases, the problem of two contacting bodies is treated with the Hertzian theory. According to this theory, the contact area is an ellipse. However, the conditions for applying the Hertzian theory to the wheel-rail contact are not fulfilled in several cases. Therefore, a more detailed model of the wheel-rail contact should be able to describe non-elliptic contact areas. There are several methods to deal with this problem: On the one hand, there are fast methods; a survey on these methods is given in [1]. Because of their comparatively low computational effort, these methods can be integrated into an MBS model, where these calculations have to be performed very often. Their disadvantage is that they only estimate the actual contact area. On the other hand, there are very precise method like the program CONTACT by Kalker [2] or Finite Element models of wheel and rail. These methods yield very accurate results, but require a high computational effort. In this paper, a method will be used which can be seen as a compromise between accuracy and computational efficiency.

\section{Vehicle-track model}

The vehicle-track model is an elastic multi-body system (EMBS). It describes a passenger coach which is running on a straight track. The bodies of which the system consists are displayed in Fig. 1.

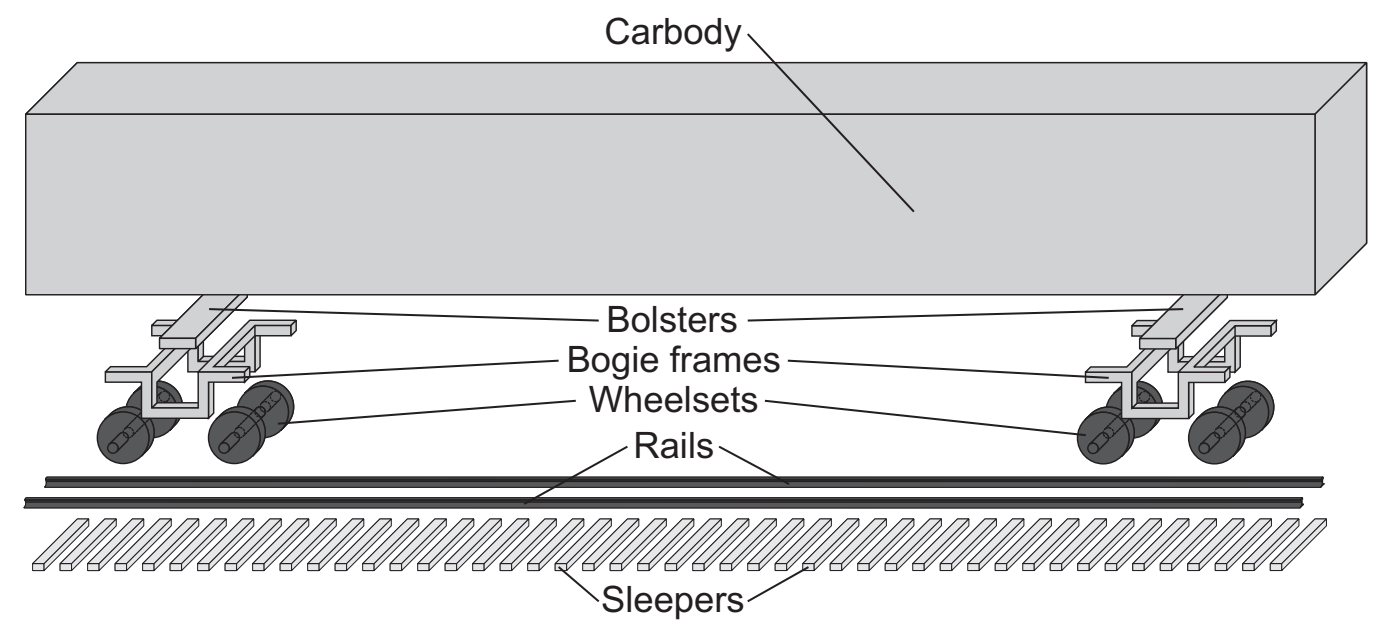

Figure 1: Bodies of the vehicle-track system. Dark bodies are modelled as elastic bodies. 
The passenger coach has two bogies, each one equipped with two wheelsets. The four wheelsets and the two rails are modelled as elastic bodies, all other bodies are considered as rigid bodies. All bodies can perform all six rigid body motions except the bolsters which can perform only yaw motions relative to the carbody. The system contains eight force elements describing the rolling contact; these elements act between the wheelsets and the rails. Furthermore, elements describing dry friction are acting between the carbody and the bolsters. All other force elements, which connect the bodies, are linear springs and dampers. The parameters of the passenger coach are taken from the works of Diepen [3] and Kim [4].

In contrast to "common" MBS-models of railway vehicles, which consist only of rigid bodies, this model is enhanced in several aspects: The wheelsets and the rails are modelled as elastic bodies, and for the wheel-rail contacts a more detailed modelling is used. These enhancements will be described in the following.

\subsection{Elastic wheelset}

Usually, the motions of an elastic body integrated into an EMBS are described by superposing the motions of the undeformed body, the so-called rigid body motions, and the deformation motions. This kinematics is displayed in Fig. 2. Details concerning EMBS can be found e.g. in [5]. The translation of the body is given by the vector

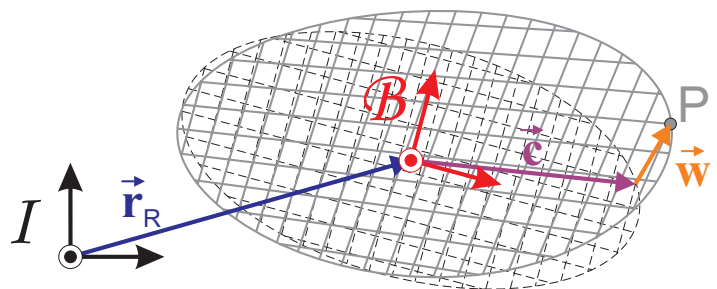

Figure 2: Kinematics of a particle of an elastic body.

$\mathbf{r}_{\mathrm{R}}^{\mathcal{I}}$ describing the position of the reference point R, e.g. the centre of gravity, in the inertial system $\mathcal{I}$. The rotation of the body is expressed by the rotation matrix $\mathcal{A}^{\mathcal{I B}}$ describing the transformation between the inertial system $\mathcal{I}$ and the body-fixed system $\mathcal{B}$. In the undeformed state, the position of a certain particle of the body is given by the vector $\mathbf{c}^{\mathcal{B}}$. Due to the deformation, the particle is shifted by the vector $\mathbf{w}^{\mathcal{B}}\left(\mathbf{c}^{\mathcal{B}}\right)$, which describes the deformation field, to its current position denoted by the point $P$. In total, the position of the particle can be expressed by:

$$
\mathbf{r}_{\mathrm{P}}^{\mathcal{I}}=\mathrm{r}_{\mathrm{R}}^{\mathcal{I}}+\mathbf{A}^{\mathcal{I B}}\left[\mathbf{c}^{\mathcal{B}}+\mathbf{w}^{\mathcal{B}}\left(\mathbf{c}^{\mathcal{B}}\right)\right]
$$

Compared with other components of a railway vehicle like the bogie frame or the car body, the wheelset performs a large rotation due to its rolling motion. However, the wheel-rail forces resulting from the contact always act at the lowest region of the wheelset. As a result, the wheel-rail contact moves around the wheelset, if it is 
observed from the frame $\mathcal{B}$ fixed to the wheelset. In other words, if $\mathbf{c}_{W R}^{\mathcal{B}}$ denotes the current position of the wheel-rail contact with respect to the body-fixed frame $\mathcal{B}$, then this vector depends on the increasing overturning angle $\chi=\chi(t)$ and thereby on the time:

$$
\mathbf{c}_{W R}^{\mathcal{B}}=\mathbf{c}_{W R}^{\mathcal{B}}(\chi(t))
$$

Usually, the deformations of an elastic body are described by a modal synthesis. Here, shape functions $\mathbf{w}_{i}(\mathbf{c})$ depending on the location $\mathbf{c}$ which are scaled by the timedependent modal coordinates $q_{i}(t)$ are superposed. Thereby, the deformation $\mathbf{u}^{\mathcal{B}}$ at the reference location $\mathbf{c}^{\mathcal{B}}$ at the time $t$ is given by:

$$
\mathbf{w}^{\mathcal{B}}\left(\mathbf{c}^{\mathcal{B}}, t\right)=\sum_{i=1}^{N} \mathbf{w}_{i}\left(\mathbf{c}^{\mathcal{B}}\right) q_{i}(t)
$$

All vectors are displayed in the body-fixed frame $\mathcal{B}$. - For the description of the rotating elastic wheelset, it is necessary to calculate the deformation at the contact point. However, as equation (2) shows, the reference location $\mathbf{c}_{W R}^{\mathcal{B}}$ of the wheel-rail contact is permanently varying. As a result, the shape functions also depend on time:

$$
\mathbf{w}^{\mathcal{B}}\left(\mathbf{c}_{W R}^{\mathcal{B}}(t), t\right)=\sum_{i=1}^{N} \mathbf{w}_{i}\left(\mathbf{c}_{W R}^{\mathcal{B}}(t)\right) q_{i}(t)
$$

Usually, the wheelset is a rotational symmetric structure. By exploiting the characteristics of such a structure, the problem described above can be solved without loss of accuracy: For a rotational symmetric structure, the description using cylindrical coordinates is obvious, namely the radial coordinate $r$, the axial coordinate $y$ and the azimut $\phi$. If linear elasticity of the material with the shear modulus $G$, Poisson's ratio $\nu$ and the density $\rho$ is assumed and the parameters are distributed in a rotational symmetric way, i.e. $G=G(r, y), \nu=\nu(r, y)$ and $\rho=\rho(r, y)$, a semi-analytic solution can be applied to the equation of motion of a three-dimensional continuum, which is e.g. given in [6]:

$$
G\left[\Delta \mathbf{w}+\frac{1}{1-2 \nu} \operatorname{grad} \operatorname{div} \mathbf{w}\right]=\rho \ddot{\mathbf{w}}
$$

It is also obvious to express the displacements in the direction of the cylindrical coordinates by using the radial deformation $R=R(r, \phi, y, t)$, the tangential deformation $T=T(r, \phi, y, t)$ and the axial deformation $V=V(r, \phi, y, t)$. If the 2-axis is the axis of the rotational symmetry, the displacement vector can be written as:

$$
\mathbf{w}^{\mathcal{B}}=\left[\begin{array}{ccc}
\cos \phi & 0 & \sin \phi \\
0 & 1 & 0 \\
-\sin \phi & 0 & \cos \phi
\end{array}\right]\left[\begin{array}{l}
T(r, \phi, y, t) \\
V(r, \phi, y, t) \\
R(r, \phi, y, t)
\end{array}\right]=\mathbf{A}_{2}(\phi) \mathbf{u}(r, \phi, y, t)
$$

As a result, the eigenmodes describing the radial deformation $R$, the tangential defor- 
mation $T$ and the axial deformation $V$ can be written as:

$$
\mathbf{u}_{i}(r, \phi, y)=\left[\begin{array}{l}
T_{i}(r, \phi, y) \\
V_{i}(r, \phi, y) \\
R_{i}(r, \phi, y)
\end{array}\right]=\underbrace{\left[\begin{array}{l}
T_{i, A}(r, y) \\
V_{i, A}(r, y) \\
R_{i, A}(r, y)
\end{array}\right]}_{\mathbf{u}_{i, A}(r, y)} \cos \left(k_{i} \phi\right)+\underbrace{\left[\begin{array}{c}
T_{i, B}(r, y) \\
V_{i, B}(r, y) \\
R_{i, B}(r, y)
\end{array}\right]}_{\mathbf{u}_{i, B}(r, y)} \sin \left(k_{i} \phi\right)
$$

The vector $\mathbf{w}_{i}(r, \phi, y)$ describing the deformation in the direction of cartesian coordinates is obtained by:

$$
\mathbf{w}_{i}(r, \phi, y)=\left[\begin{array}{c}
U_{i}(r, \phi, y) \\
V_{i}(r, \phi, y) \\
W_{i}(r, \phi, y)
\end{array}\right]=\mathbf{A}_{2}(\phi) \mathbf{u}_{i}(r, \phi, y)
$$

It is important to note that each eigenmode has one and only one periodicity $k_{i}$ being an integer. For $k_{i} \neq 0$, double eigenfrequencies occur belonging to two orthogonal modes $\mathbf{u}_{i, 1}$ and $\mathbf{u}_{i, 2}$, which can be written as:

$$
\begin{aligned}
& \mathbf{u}_{i, 1}(r, \phi, y)=\mathbf{u}_{i, A}(r, y) \cos \left(k_{i} \phi\right)+\mathbf{u}_{i, B}(r, y) \sin \left(k_{i} \phi\right) \\
& \mathbf{u}_{i, 2}(r, \phi, y)=\mathbf{u}_{i, A}(r, y) \sin \left(k_{i} \phi\right)-\mathbf{u}_{i, B}(r, y) \cos \left(k_{i} \phi\right)
\end{aligned}
$$

If both double eigenmodes are taken into account for the modal synthesis, the deformation vector $\mathbf{u}$ can be expressed as:

$$
\mathbf{u}(r, \phi, y, t)=\sum_{i}\left[\mathbf{u}_{i, 1}(r, \phi, y) q_{i, 1}(t)+\mathbf{u}_{i, 2}(r, \phi, y) q_{i, 2}(t)\right]
$$

An intermediate, axle-fixed frame $\mathcal{A}$ is now introduced, as displayed in Fig. 3. This

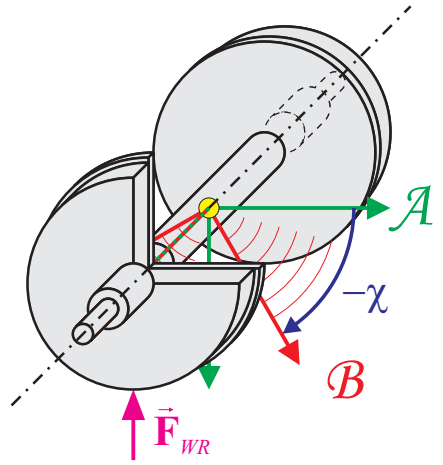

Figure 3: Body-fixed frame $\mathcal{B}$ and intermediate axle-fixed frame $\mathcal{A}$ of the wheelset.

frame performs all motions of the body-fixed frame $\mathcal{B}$ except the overturning motion $\chi=\chi(t)$. Thereby, the wheel-rail force $\overrightarrow{\mathbf{F}}_{W R}$ doesn't move around the wheel in this 
frame. The relation between the new frame $\mathcal{A}$ and the body-fixed frame $\mathcal{B}$ is expressed by the matrix $\mathbf{A}^{\mathcal{A B}}$ :

$$
\mathbf{A}^{\mathcal{A B}}=\left[\begin{array}{ccc}
\cos \chi & 0 & \sin \chi \\
0 & 1 & 0 \\
-\sin \chi & 0 & \cos \chi
\end{array}\right]=\mathbf{A}_{2}(\chi)
$$

The deformation in the new frame $\mathcal{A}$ can be described as follows:

$$
\mathbf{w}^{\mathcal{A}}=\mathbf{A}^{\mathcal{A B}} \mathbf{w}^{\mathcal{B}}=\mathbf{A}_{2}(\chi) \mathbf{A}_{2}(\phi) \mathbf{u}=\mathbf{A}_{2}(\phi+\chi) \mathbf{u}
$$

The sum of the azimut $\phi$ in the frame $\mathcal{B}$ and the overturning angle $\chi$ is the azimut in the intermediate frame $\mathcal{A}$ which is denoted with $\theta$ :

$$
\theta=\phi+\chi \Leftrightarrow \phi=\theta-\chi
$$

In the frame $\mathcal{A}$, the position of the wheel-rail contact is described by a constant value of $\theta$. It should be pointed out, that the coordinate $\theta$ is a local coordinate denoting a location in space in contrast to the material coordinate $\phi$ denoting a particle.

To describe the deformation in the frame $\mathcal{A}$, the deformation has to be expressed depending on $\theta$. By inserting the relation (14) into the descriptions (9) and (10) of the eigenmodes, the following expressions are obtained.

$$
\begin{aligned}
& \mathbf{u}_{i, 1}(r, \phi, y)=\mathbf{u}_{i, 1}(r, \theta, y) \cos \left(k_{i} \chi\right)+\mathbf{u}_{i, 2}(r, \theta, y) \sin \left(k_{i} \chi\right) \\
& \mathbf{u}_{i, 2}(r, \phi, y)=\mathbf{u}_{i, 2}(r, \theta, y) \cos \left(k_{i} \chi\right)-\mathbf{u}_{i, 1}(r, \theta, y) \sin \left(k_{i} \chi\right)
\end{aligned}
$$

The superposition of the two eigenmodes, which are scaled with their modal coordinates $q_{i, 1}$ and $q_{i, 2}$, leads to

$$
\begin{aligned}
\mathbf{u}_{i, 1}( & r, \phi, y) q_{i, 1}(t)+\mathbf{u}_{i, 2}(r, \phi, y) q_{i, 2}(t) \\
= & {\left[\mathbf{u}_{i, 1}(r, \theta, y) \cos \left(k_{i} \chi\right)+\mathbf{u}_{i, 2}(r, \theta, y) \sin \left(k_{i} \chi\right)\right] q_{i, 1}(t) } \\
& +\left[\mathbf{u}_{i, 2}(r, \theta, y) \cos \left(k_{i} \chi\right)-\mathbf{u}_{i, 1}(r, \theta, y) \sin \left(k_{i} \chi\right)\right] q_{i, 2}(t) \\
= & \mathbf{u}_{i, 1}(r, \theta, y)\left[q_{i, 1}(t) \cos \left(k_{i} \chi\right)-q_{i, 2}(t) \sin \left(k_{i} \chi\right)\right] \\
& +\mathbf{u}_{i, 2}(r, \theta, y)\left[q_{i, 1}(t) \sin \left(k_{i} \chi\right)+q_{i, 2}(t) \cos \left(k_{i} \chi\right)\right]
\end{aligned}
$$

The expressions in the brackets can be defined as the new modal coordinates $q_{i, 1}^{*}$ and $q_{i, 2}^{*}$ :

$$
\begin{aligned}
& q_{i, 1}^{*}(t)=q_{i, 1}(t) \cos \left(k_{i} \chi\right)-q_{i, 2}(t) \sin \left(k_{i} \chi\right) \\
& q_{i, 2}^{*}(t)=q_{i, 1}(t) \sin \left(k_{i} \chi\right)+q_{i, 2}(t) \cos \left(k_{i} \chi\right)
\end{aligned}
$$

As a result, the modal synthesis for the deformation depending on the new azimut $\theta$ and the new modal coordinates $q_{i, 1}^{*}$ and $q_{i, 2}^{*}$ is obtained:

$$
\mathbf{u}(r, \theta, y, t)=\sum_{i}\left[\mathbf{u}_{i, 1}(r, \theta, y) q_{i, 1}^{*}(t)+\mathbf{u}_{i, 2}(r, \theta, y) q_{i, 2}^{*}(t)\right]
$$


To determine the velocity of the deformation, it has to be taken into account that the coordinate $\theta$ is a local coordinate, not a material one. Therefore, also the vectors $\mathbf{u}_{i, 1}(r, \theta, y)$ and $\mathbf{u}_{i, 2}(r, \theta, y)$ have to be derived with respect to time:

$$
\begin{aligned}
\dot{\mathbf{u}}(r, \theta, y, t)=\sum_{i}[ & \frac{\mathrm{d} \mathbf{u}_{i, 1}(r, \theta, y)}{\mathrm{d} t} q_{i, 1}^{*}(t)+\mathbf{u}_{i, 1}(r, \theta, y) \dot{q}_{i, 1}^{*}(t) \\
& \left.+\frac{\mathrm{d} \mathbf{u}_{i, 2}(r, \theta, y)}{\mathrm{d} t} q_{i, 2}^{*}(t)+\mathbf{u}_{i, 2}(r, \theta, y) \dot{q}_{i, 2}^{*}(t)\right]
\end{aligned}
$$

By considering the relation (14) and the structure of the eigenmodes (9) and (10), the following relations are found:

$$
\begin{aligned}
& \frac{\mathrm{d} \mathbf{u}_{i, 1}(r, \theta, y)}{\mathrm{d} t}=\frac{\partial \mathbf{u}_{i, 1}}{\partial \theta} \frac{\mathrm{d} \theta}{\mathrm{d} t}=-k_{i} \mathbf{u}_{i, 2}(r, \theta, y)(-\dot{\chi})=\mathbf{u}_{i, 2}(r, \theta, y) k_{i} \dot{\chi} \\
& \frac{\mathrm{d} \mathbf{u}_{i, 2}(r, \theta, y)}{\mathrm{d} t}=\frac{\partial \mathbf{u}_{i, 2}}{\partial \theta} \frac{\mathrm{d} \theta}{\mathrm{d} t}=k_{i} \mathbf{u}_{i, 1}(r, \theta, y)(-\dot{\chi})=-\mathbf{u}_{i, 1}(r, \theta, y) k_{i} \dot{\chi}
\end{aligned}
$$

Inserting these relations into (20) and factoring out the vectors $\mathbf{u}_{i, 1}(r, \theta, y)$ and $\mathbf{u}_{i, 2}(r, \theta, y)$ leads to the expression

$$
\begin{aligned}
\dot{\mathbf{u}}(r, \theta, y, t)=\sum_{i}[ & \mathbf{u}_{i, 1}(r, \theta, y)\left[\dot{q}_{i, 1}^{*}(t)-k_{i} \dot{\chi} q_{i, 2}^{*}(t)\right] \\
& \left.+\mathbf{u}_{i, 2}(r, \theta, y)\left[\dot{q}_{i, 2}^{*}(t)+k_{i} \dot{\chi} q_{i, 1}^{*}(t)\right]\right]
\end{aligned}
$$

Now, the deformation observed in the frame $\mathcal{A}$ can be described. The modal synthesis (19) is inserted into (13), and the relation (8) is used. As a result, the following expression is obtained:

$$
\begin{aligned}
\mathbf{w}^{\mathcal{A}}(r, \theta, y, t) & =\mathbf{A}_{2}(\theta) \mathbf{u}(r, \theta, y, t) \\
& =\sum_{i}\left[\mathbf{A}_{2}(\theta) \mathbf{u}_{i, 1}(r, \theta, y) q_{i, 1}^{*}(t)+\mathbf{A}_{2}(\theta) \mathbf{u}_{i, 2}(r, \theta, y) q_{i, 2}^{*}(t)\right] \\
& =\sum_{i}\left[\mathbf{w}_{i, 1}(r, \theta, y) q_{i, 1}^{*}(t)+\mathbf{w}_{i, 2}(r, \theta, y) q_{i, 2}^{*}(t)\right]
\end{aligned}
$$

In an analogous way, the velocity of the deformation observed in the frame $\mathcal{A}$ is obtained:

$$
\begin{aligned}
\stackrel{\mathbf{w}}{\mathcal{A}}^{\mathcal{A}}(r, \theta, y, t)= & \mathbf{A}_{2}(\theta) \dot{\mathbf{u}}(r, \theta, y, t) \\
= & \sum_{i}\left[\mathbf{w}_{i, 1}(r, \theta, y)\left[\dot{q}_{i, 1}^{*}(t)-k_{i} \dot{\chi} q_{i, 2}^{*}(t)\right]\right. \\
& \left.\quad+\mathbf{w}_{i, 2}(r, \theta, y)\left[\dot{q}_{i, 2}^{*}(t)+k_{i} \dot{\chi} q_{i, 1}^{*}(t)\right]\right]
\end{aligned}
$$

The star indicates that the vector ${ }_{\mathbf{w}}^{\mathcal{A}}(r, \theta, y, t)$ is the deformational velocity observed in the frame $\mathcal{A}$. The relative velocity of the particle is given by:

$$
\dot{\mathbf{w}}^{\mathcal{A}}(r, \theta, y, t)=\mathbf{w}^{*} \mathcal{A}(r, \theta, y, t)+\boldsymbol{\omega}_{\mathcal{A B}}^{\mathcal{A}} \times \mathbf{w}^{\mathcal{A}}(r, \theta, y, t), \boldsymbol{\omega}_{\mathcal{A B}}^{\mathcal{A}}=\left[\begin{array}{lll}
0 & \dot{\chi} & 0
\end{array}\right]^{T}
$$


In this formulation, a constant value of $\theta$ describing the position of the wheel-rail contact in the frame $\mathcal{A}$ can be set. This means that the angle $\phi$ describing a particle is chosen in such a way, that the relation

$$
\theta=\phi(t)+\chi(t)=\text { const. }
$$

is always fulfilled. As a consequence, the particle which is observed changes over time. All in all, this method provides a simple and accurate way to describe the acting of the wheel-rail forces moving around the wheelsets.

\subsection{Elastic track}

The track model is displayed in Fig. 4. It is based on the track model by Ripke [7], but uses an enhanced model of the rail. The model consists of two elastic rails, which

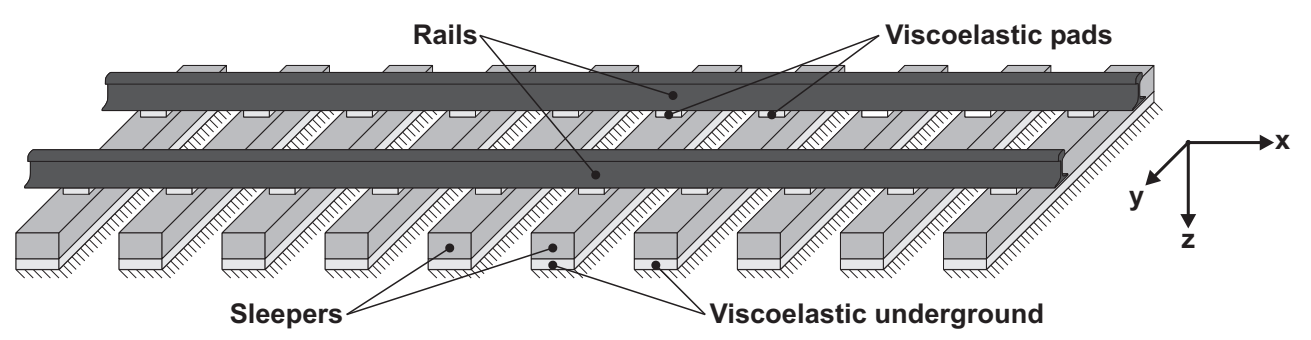

Figure 4: Model of the track containing elastic rails.

are supported by discrete sleepers. The sleepers are considered as rigid bodies, which can perform all six motions. They are connected to the rails by visco-elastic layers reprensenting the pads and are supported by visco-elastic layers representing the underground. The inclination of the rails towards the middle of the track is taken into account in this model. A value of 1/40 is used; this value is the standard in Germany, Austria, Switzerland and other countries. For the rails, the profile UIC60 is chosen, which is widely used in Europe.

A general problem arising in the modelling of the track is that the vehicle reaches the end of the track very soon if it is running with high speeds. To circumvent this problem, the same boundary conditions are applied at the ends of the track. Thereby, the track forms a ring, but the curvature of the ring is neglected. Of course, it has to be checked out how long the ring has to be to show approximatively the same dynamic behaviour as a very long track. In this case, comparisons showed that a model using 128 sleepers is sufficient. With a sleeper spacing of $\Delta x_{\mathrm{S}}=0.6 \mathrm{~m}$ the track has a total length of $l_{\mathrm{R}}=76.8 \mathrm{~m}$.

The foot of the rail is connected to the sleepers, lateral wheel-rail forces act at the rail head, and the web of the rail is comparatively thin. Therefore, deformations of the cross-section should be taken into account. This cannot be done by applying a common beam theory, since these theories assume that the cross-section remains undeformed. Therefore, the rail is modelled as a three-dimensional continuum. The rail 
has a prismatic shape, which can be exploited for a semi-analytic solution of the equation (5). In contrast to the wheelset, the deformations are described in the directions of cartesian coordinates. The longitudinal coordinate $x$ is separated from the other coordinates $y$ and $z$. Therefore, the shape functions of the rail can be expressed as:

$$
\mathbf{w}_{i, \mathrm{R}}=\left[\begin{array}{c}
U_{i}(y, z) \sin \left(\kappa_{i} x+\beta_{i}\right) \\
V_{i}(y, z) \cos \left(\kappa_{i} x+\beta_{i}\right) \\
W_{i}(y, z) \cos \left(\kappa_{i} x+\beta_{i}\right)
\end{array}\right], \kappa_{i}=c_{i} \frac{2 \pi}{l_{\mathrm{R}}}, c_{i} \in \mathbb{Z}
$$

The eigenmodes, which are obtained, describe the usual bending motions as well as deformations of the cross-section, as displayed in Fig. 5.

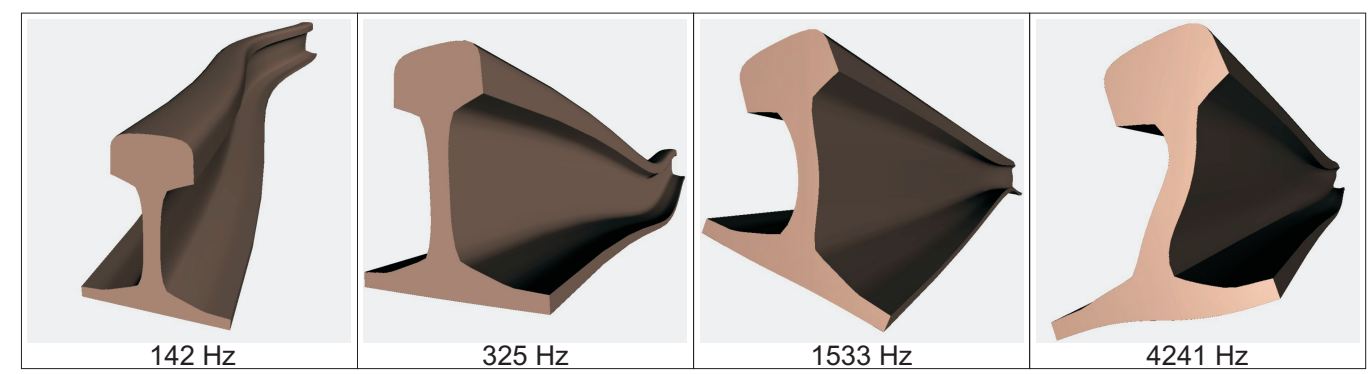

Figure 5: Eigenmodes of the rail, wavelength $l_{\mathrm{R}} / c_{i}=2.4 \mathrm{~m}$

Due to the periodic support of the rails by the discrete sleepers, an eigenmode of the entire track cannot be characterised by only one wavenumber $\kappa_{i}$. However, the structure of the eigenmodes according to (28) gives a continuous distribution of the deformations depending on the longitudinal coordinate $x$, so that the motion of the wheel-rail forces along the rail can be taken into account in a comparatively easy way.

Since a completely rigid track leads to unrealistic simulation results, a very simple track subsystem as displayed in Fig. 6 is used for comparison. It consists of a rigid

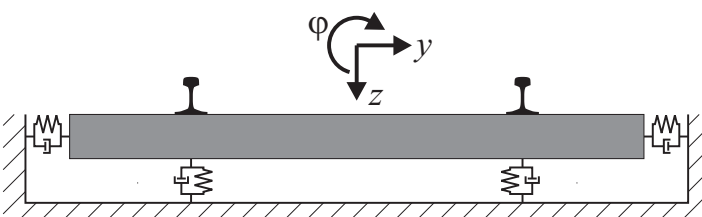

Figure 6: Simple track model

body, which can perform lateral motions $y$, vertical motions $z$ and roll motions $\varphi$. Each wheelset is supported by one of such subsystems. This model will be referenced with "rigid rails" in contrast to the detailed track model displayed in Fig. 4, which will be referenced with "elastic rails". 


\subsection{Wheel-rail contact}

From the point of view of multi-body dynamics, the wheel-rail contact is a force element, i.e. its inputs and outputs are the relative kinematics of wheel and rail and the resulting forces and torques, respectively. These forces result from stresses acting in the contact patch which are related to the deformations. For the integration of the wheel-rail contact into an EMBS, especially for wheelsets and rails modelled as flexible bodies, it is useful to split up the deformations into global deformations and local deformations. Global deformations refer to deformations concerning the entire structure, e.g. bending of the wheelset's axle or of the rail. Local deformations refer to deformations in the contact area and its immediate neighbourhood.

The first step is the geometric analysis of the contact. To simplify this step, it is assumed that the profiles move due to the global deformations of wheelset and rail, but remain undeformed. This assumption is justified by the fact that the wheel rim and the railhead are comparatively massive parts. Thereby, the kinematics of the two profiles can be determined by the kinematics of two nodes, the one belonging to the wheelset, the other one belonging to the rail. By considering the profile of the rail and the enveloppe of the wheel, two intersection points $\mathrm{P}_{L}$ and $\mathrm{P}_{R}$ are found, as displayed in Fig. 7. As a result, the fields of interpenetration $\delta(x, y)$ and the relative velocities
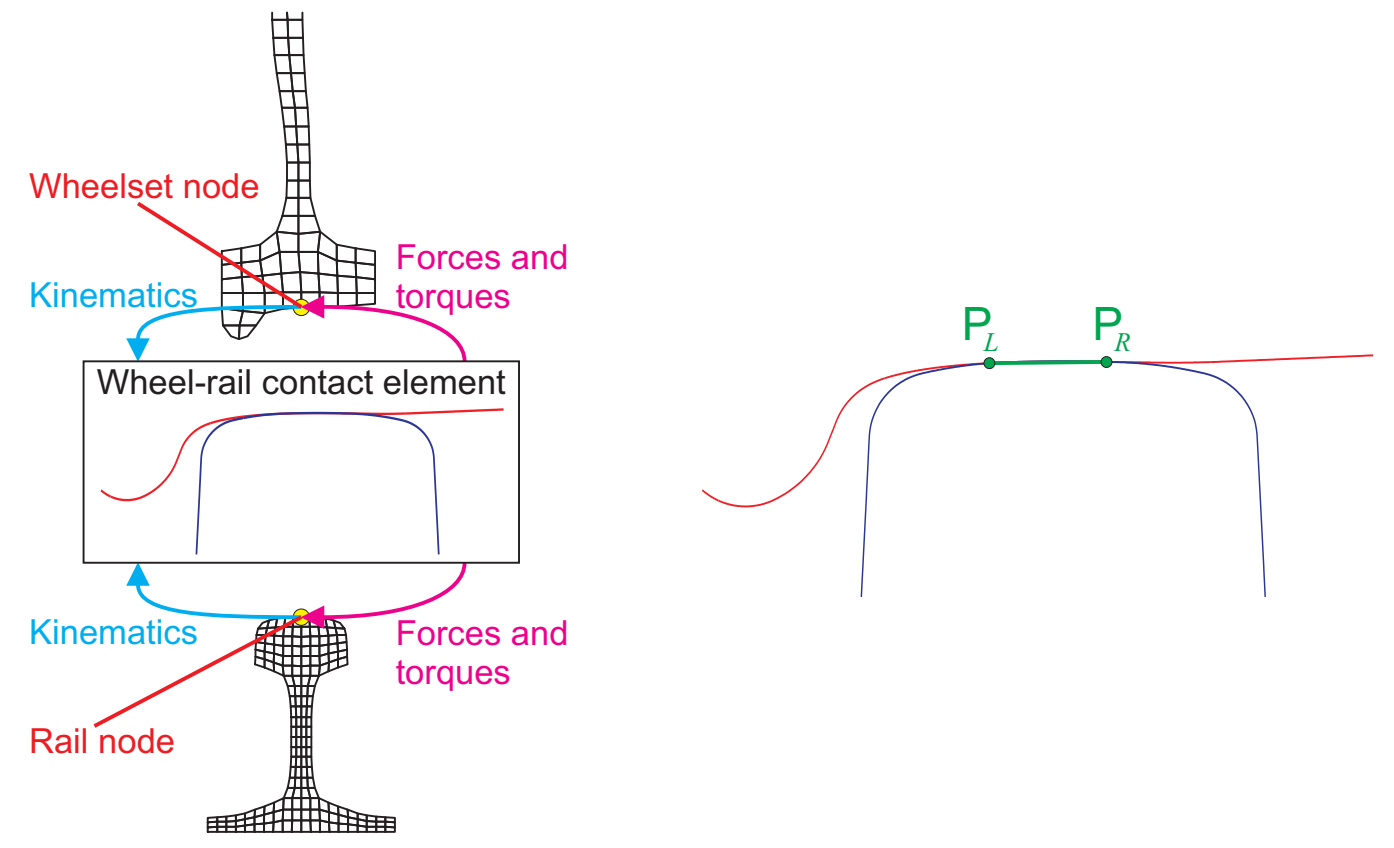

Figure 7: Left: Coupling of the wheel-rail contact element to the elastic wheelset and the elastic rail. Right: Determination of the contact plain.

$v_{1,0}(x, y)$ and $v_{2,0}(x, y)$ are obtained. The plain in which the interpenetration and the velocities are described is defined by the longitudinal coordinate $x$ of the rail and the chord which connects the points $\mathrm{P}_{L}$ and $\mathrm{P}_{R}$. It should be mentioned, that the nodes 
don't necessarily have to lie in the actual contact area, because the shifting of the contact area is performed internally in the contact. The effect of the shifting of the contact is taken into account by additional torques.

Naturally, the solid bodies cannot interpenetrate each other, but a deformation $w(x, y)$ occurs which compensates the interpenetration in the actual contact. Within the contact area, the normal pressure $p(x, y)$ is positive. Outside the contact area, the pressure vanishes. Therefore, two conditions can be formulated:

$$
\begin{aligned}
& \delta(x, y)-w(x, y)=0 \wedge p(x, y)>0 \quad \text { inside the contact area } \\
& \delta(x, y)-w(x, y)<0 \wedge p(x, y)=0 \quad \text { outside the contact area }
\end{aligned}
$$

The complete theoretical base for the contact mechanics is given by Kalker in [2]. Since the contact area is small compared to the main dimensions of wheel and rail, the contacting bodies can be considered as elastic half-spaces. The equations of Boussinesq and Cerrutti give the relation between the stresses acting on the surfaces of the half-space and the deformations occurring at the surfaces. Since in the case of the wheel-rail contact both contacting bodies consist of steel, equal material parameters, i.e. the shear modulus $G$ and Poisson's ratio $\nu$, can be assumed for both halfspaces. As a result, the relations between the tangential stresses $\tau_{1}$ and $\tau_{2}$ and the tangential deformations $u_{1}$ and $u_{2}$ on the one hand and the relation between the pressure $p$ and the normal deformation $w$ on the other hand are decoupled. Therefore, the equations of Boussinesq and Cerrutti can be written as:

$$
\begin{aligned}
u_{1}(X, Y)= & \frac{1}{\pi G} \int_{A}\left[\frac{1-\nu}{R}+\frac{(X-x)^{2} \nu}{R^{3}}\right] \tau_{1}(x, y) \mathrm{d} A \\
& +\frac{\nu}{\pi G} \int_{A} \frac{(X-x)(Y-y)}{R^{3}} \tau_{2}(x, y) \mathrm{d} A \\
u_{2}(X, Y)= & \frac{\nu}{\pi G} \int_{A} \frac{(X-x)(Y-y)}{R^{3}} \tau_{1}(x, y) \mathrm{d} A \\
& +\frac{1}{\pi G} \int_{A}\left[\frac{1-\nu}{R}+\frac{(Y-y)^{2} \nu}{R^{3}}\right] \tau_{2}(x, y) \mathrm{d} A \\
w(X, Y)= & \frac{1-\nu}{\pi G} \int_{A} \frac{p(x, y)}{R} \mathrm{~d} A, \quad R=\sqrt{(X-x)^{2}+(Y-y)^{2}} .
\end{aligned}
$$

Since the equation (33) is decoupled from the equations (31) and (32), the normal contact problem, i.e. the determination of the pressure field $p(x, y)$ can be solved as the second step of the contact analysis, following the first step of the geometric analysis. The third step is the solution of the tangential contact, i.e. calculating the distributions $\tau_{1}(x, y)$ and $\tau_{2}(x, y)$ of the tangential stresses.

For the solution, the problem is discretised by using a grid with the constant $\Delta a$, where the stresses and deformations are only considered at the nodes of the grid. The stresses are expressed by superposing local bilinear functions $f_{k}(x, y)$ which are 1 at 
the point $\left\langle x_{k}, y_{k}\right\rangle$ and 0 at all other points of the grid, as shown in Fig. 8:

$$
\begin{aligned}
\tau_{1}(x, y) & =\sum_{k} \tau_{1}\left(x_{k}, y_{k}\right) f_{k}(x, y)=\sum_{k} \tau_{1, k} f_{k}(x, y), \\
\tau_{2}(x, y) & =\sum_{k} \tau_{2}\left(x_{k}, y_{k}\right) f_{k}(x, y)=\sum_{k} \tau_{2, k} f_{k}(x, y), \\
p(x, y) & =\sum_{k} p\left(x_{k}, y_{k}\right) f_{k}(x, y)=\sum_{k} p_{k} f_{k}(x, y) .
\end{aligned}
$$
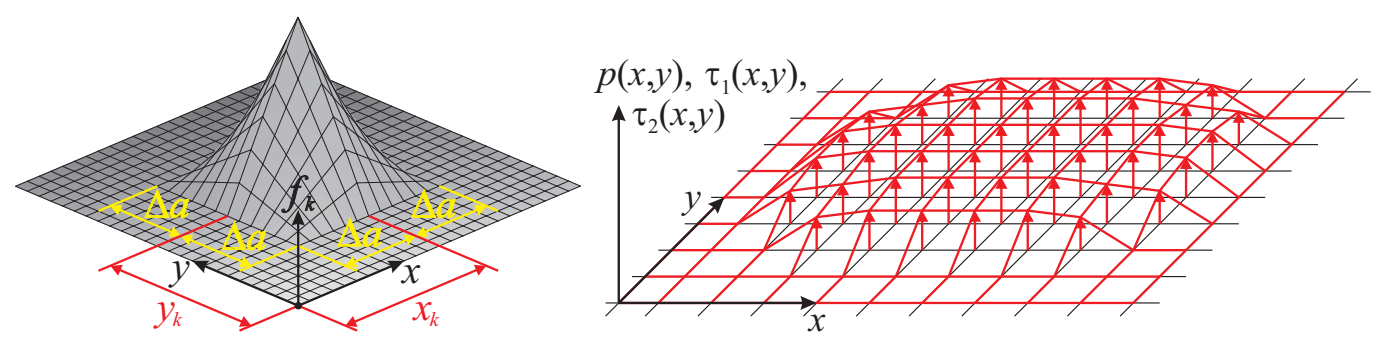

Figure 8: Local bilinear function $f_{k}$ (left), discretisation of the distribution of the stresses (right)

By inserting the discretised distributions of $\tau_{1}(x, y), \tau_{2}(x, y)$ and $p(x, y)$ into the Boussinesq-Cerrutti equations, two systems of linear equations are obtained, one for the normal contact problem, the other for the tangential contact problem:

$$
\mathbf{H}_{33} \mathbf{f}_{3}=\mathbf{u}_{3},\left[\begin{array}{ll}
\mathbf{H}_{11} & \mathbf{H}_{12} \\
\mathbf{H}_{12} & \mathbf{H}_{22}
\end{array}\right]\left[\begin{array}{l}
\mathbf{f}_{1} \\
\mathbf{f}_{2}
\end{array}\right]=\left[\begin{array}{l}
\mathbf{u}_{1} \\
\mathbf{u}_{2}
\end{array}\right] .
$$

The vectors $\mathbf{f}_{1}, \mathbf{f}_{2}$ and $\mathbf{f}_{3}$ contain the stresses $\tau_{1}\left(x_{k}, y_{k}\right), \tau_{2}\left(x_{k}, y_{k}\right)$ and $p\left(x_{k}, y_{k}\right)$, respectively, at the nodes of the grid. The vectors $\mathbf{u}_{1}, \mathbf{u}_{2}$ and $\mathbf{u}_{3}$ contain the deformations $u_{1}\left(x_{i}, y_{i}\right), u_{2}\left(x_{i}, y_{i}\right)$, and $u_{3}\left(x_{i}, y_{i}\right)$ at the nodes.

Considering the normal contact problem, the main difficulty is that it is unknown at the start how many points belong to the actual contact area and, thereby, of how many equations the system actually consists. Therefore, the direct solution of the system of linear equations is not sensible, because the decomposition of the matrix requires a high computational effort. A more efficient way is the application of an iterative solution of the system, e.g. by the Gauss-Seidel method, as it has already used by Vollebregt [8] in contact mechanics. If $i$-th line of the system $\mathbf{H}_{33} \mathbf{f}_{3}=\mathbf{u}_{3}$ reads:

$$
\sum_{j=1}^{n} H_{i j}^{(33)} p_{j}=\delta_{i}, p_{j}=p\left(x_{j}, y_{j}\right), \delta_{i}=\delta\left(x_{i}, y_{i}\right)
$$

and $p_{i}^{(k)}$ is the $k$-th approximation of $p_{i}$, then the new value $p_{i}^{(k+1)}$ is obtained by:

$$
p_{i}^{(k+1)}=\frac{1}{H_{i i}^{(33)}}\left[\delta_{i}-\sum_{j=1}^{i-1} H_{i j}^{(33)} p_{j}^{(k+1)}-\sum_{j=i+1}^{n} H_{i j}^{(33)} p_{j}^{(k)}\right]
$$


This iterative scheme is performed until all differences $p_{i}^{(k+1)}-p_{i}^{(k)}$ are below a certain tolerance. It is obvious that additional conditions can be introduced easily into the scheme: If the iteration yields a negative value $p_{i}^{(k+1)}<0$, the new value is set to $p_{i}^{(k+1)}=0$.

To formulate the condition for the solution of the tangential contact problem, the actual relative velocities $v_{1, \text { rel }}(x, y)$ and $v_{2, \text { rel }}(x, y)$ in the contact have to be considered. This velocities consist of the applied relative velocities $v_{1,0}(x, y)$ and $v_{2,0}(x, y)$ of the undeformed half-spaces, which are obtained from the geometric analysis, and the deformational velocities $v_{1, d e f}(x, y)$ and $v_{2, d e f}(x, y)$ :

$$
v_{1, r e l}(x, y)=v_{1,0}(x, y)+v_{1, \text { def }}(x, y), v_{2, r e l}(x, y)=v_{2,0}(x, y)+v_{2, \text { def }}(x, y)
$$

In the case of adhesion, the relative velocities $v_{1, r e l}(x, y)$ and $v_{2, r e l}(x, y)$ vanish, and the resulting tangential stress is smaller than or equal to the maximum transmittable stress $\tau_{\text {max }}$, which is determined by the pressure $p(x, y)$ and the friction coefficient:

$$
v_{1, r e l}(x, y)=0 \wedge v_{2, r e l}(x, y)=0 \wedge \sqrt{\tau_{1}(x, y)^{2}+\tau_{2}(x, y)^{2}} \leq \tau_{\max }=\mu p(x, y)
$$

In the case of sliding, the tangential stresses act in the opposite direction of the relative velocities. This condition can be formulated in the following way:

$$
\tau_{1}(x, y) v_{2, r e l}(x, y)-\tau_{2}(x, y) v_{1, r e l}(x, y)=0 \wedge \sqrt{\tau_{1}(x, y)^{2}+\tau_{2}(x, y)^{2}}=\mu p(x, y)
$$

If the deformation field at a small time-step $\Delta t$ before the current time is given by $u_{1}^{*}(x, y)$ and $u_{2}^{*}(x, y)$, then the deformational velocities can be approximated by:

$$
v_{1, d e f}(x, y) \approx \frac{u_{1}(x, y)-u_{1}^{*}(x, y)}{\Delta t}, v_{2, d e f}(x, y) \approx \frac{u_{2}(x, y)-u_{2}^{*}(x, y)}{\Delta t}
$$

As Vollebregt showed in [8], the Gauss-Seidel method is an efficient way to solve the system of linear equations (37) and taking into account the non-linear conditions (42).

\section{Calculation results}

To investigate the impact of the structural elasticities on the wheel-rail contact, two models with different configurations are used: In the first model, the wheelsets are considered as rigid bodies and the simple track model displayed in Fig. 6 is used. In the second model, the structural dynamics of the wheelsets are taken into account; all eigenmodes of the wheelset, which belong to eigenfrequencies below $5000 \mathrm{~Hz}$ are used as shape functions for the modal synthesis. Furthermore, the detailed track model including elastic rails, which is shown in Fig. 4, is used.

For the wheel, the profile S1002 is chosen. As already mentioned, the rail profile UIC60 is used. This profile combination is widely used in Europe. Furthermore, an inclination of $1 / 40$ for the rails is assumed; this value is the standard in Germany, 
Austria, Switzerland and other countries. From earlier research, cp. [1], it is known, that non-elliptic contact patches can occur for this profile combination; such patches cannot be described exactly with the Hertzian theory, which assumes that the contact area is an ellipse. For the calculation of the stresses, the constant of the grid is set to $\Delta a=0.75 \mathrm{~mm}$. - Generally, the contact at the right wheel of the vehicle's front wheelset is considered in the following investigation.

\subsection{Centred running}

The first scenario which is investigated is the centred, undisturbed running of the wheelset. If the wheelsets and the rails are considered as rigid bodies, a stationary state is obtained and the wheel-rail forces are constant. For the case of elastic wheelsets on elastic rails, the wheel-rail forces are fluctuating due to the support of the rails by the discrete sleepers. However, as Fig.9 shows, the oscillation of the vertical force $F_{3}$ is quite small.

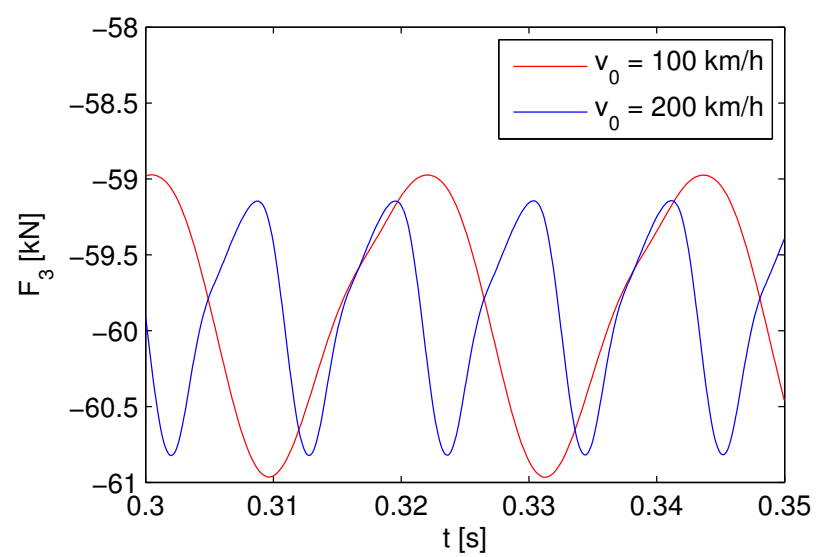

Figure 9: Time history of the vertical wheel-rail force $F_{3}$ for elastic wheelsets on elastic rails at $v_{0}=100 \mathrm{~km} / \mathrm{h}$ and $v_{0}=200 \mathrm{~km} / \mathrm{h}$

The frequency is the ratio of the driving speed $v_{0}$ and the sleeper spacing $\Delta x_{\mathrm{S}}$ :

$$
f=\frac{v_{0}}{\Delta x_{\mathrm{S}}}
$$

Using a sleeper spacing of $\Delta x_{\mathrm{S}}=0.6 \mathrm{~m}$, the frequency is $f=46.3 \mathrm{~Hz}$ for $v_{0}=$ $100 \mathrm{~km} / \mathrm{h}$ and $f=92.6 \mathrm{~Hz}$ for $v_{0}=200 \mathrm{~km} / \mathrm{h}$.

The comparison of the normal stresses occurring in the contact at the right wheel of the vehicle's front wheelset are displayed in Fig.10. On the right hand side, the areal distribution of the normal pressure is shown. The wheel is running in positive $x$-direction. On the left hand side, the relative position of the profiles of wheel and rail and the maximum pressure are displayed. 
Rigid wheelsets, rigid rails

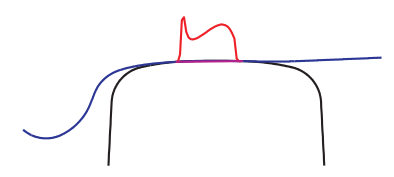

Elastic wheelsets, elastic rails

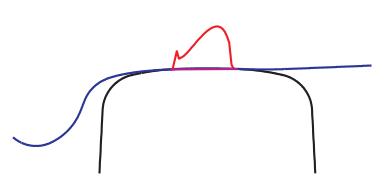

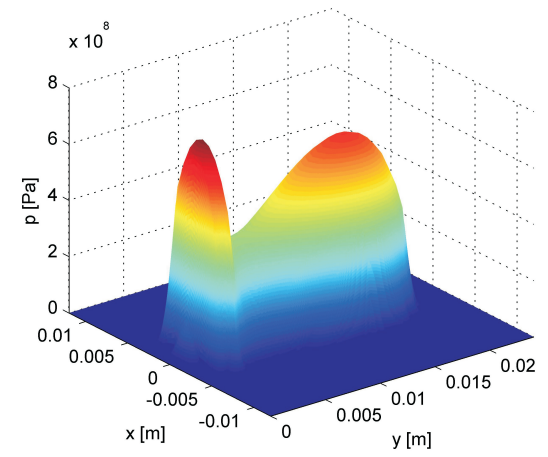

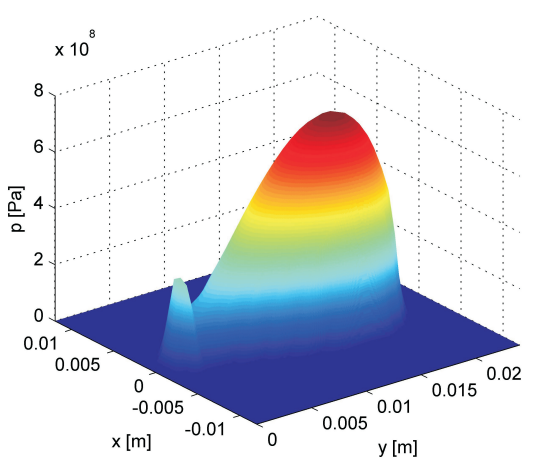

Figure 10: Distribution of the normal stresses for $v_{0}=200 \mathrm{~km} / \mathrm{h}$ and $\mu=0.3$.

Although the change of the relative kinematics of the profiles is hardly visible, the distribution of the normal pressure and thereby the contact patch is distinctly changed. The left maximum, which is very distinctive for the case of rigid wheelsets and rails, shrinks while the right maximum increases. This effect can be explained by considering the external forces, as displayed in a simplified way in Fig.11. In the case of centred running, the bearing forces $F_{B}$ at the external journals and the wheel-rail forces $F_{W R}$ are mainly acting in the vertical direction. This causes particularly a bending of the wheelset's axle. As a result, the wheel rims incline outwards. Thereby, the normal load is shifted to the right maximum, as displayed in Fig.10.

Due to the inclination of the running surface, the vector of the angular velocity resulting from the wheelset's overturning motion is not orthogonal to the running surface. As a result, a spin occurs in the contact patch. This spin can clearly be seen in the distributions of the tangential stresses which are shown in Fig.12. As already mentioned, the wheel is running in the positive $x$-direction. At the rear edge of the contact patch, an area of sliding can be recognized. The single points indicating a sliding at the front edge result from discretisation errors.

For the wear, the power dissipated due to the friction is of interest. Therefore, the distribution of the density of the frictional power $P_{F} / A$ is displayed in Fig.13. Of course, the frictional power vanishes in the zone of adhesion. Only in the zone of sliding, power can be dissipated by friction. 


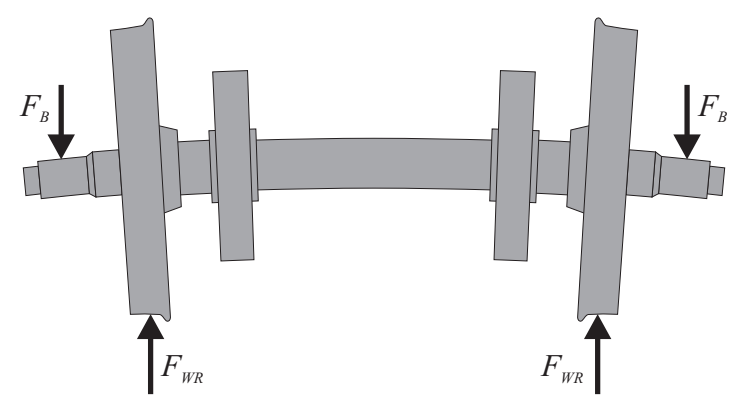

Figure 11: Bending of the wheelset due to bearing forces $F_{B}$ and wheel-rail forces $F_{W R}$
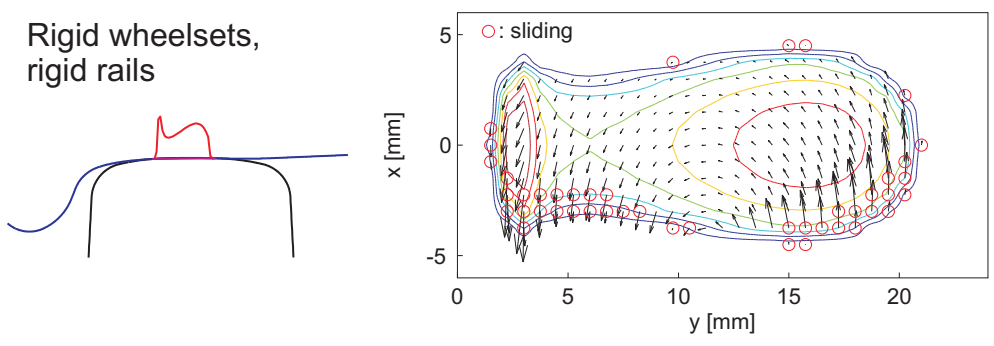

Elastic wheelsets, elastic rails
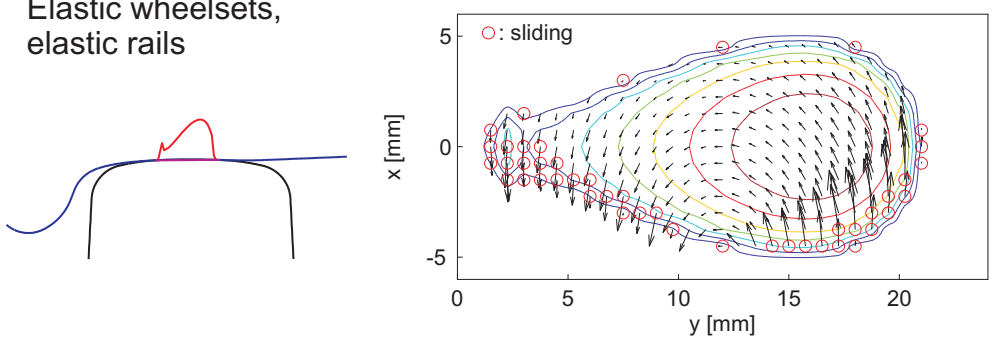

Figure 12: Distribution of the tangential stresses for $v_{0}=200 \mathrm{~km} / \mathrm{h}$ and $\mu=0.3$.

It can clearly be seen that the two distributions differ distinctly from each other. For the modelling using rigid wheelsets and rigid rails, the maximum density occurs at the left part of the contact patch. Here, the largest normal pressure occurs, which causes also high tangential stresses $\tau=\mu p$. Since the left maximum of the normal pressure shrinks drastically in the case of elastic wheelsets and elastic rails, only low values of the frictional power density can be found in the left part of the contact patch. It is remarkable that the power density reaches higher values in the case of rigid wheelsets and rigid rails. In the left part, the absolute maximum of $P_{F} / A=13 \mathrm{~W} / \mathrm{mm}^{2}$ is reached. In the right part, a relative maximum of $P_{F}=7 \mathrm{~W} / \mathrm{mm}^{2}$ occurs. In contrast, for the modelling using elastic wheelsets and elastic rails, a maximum of only $P_{F} / A=$ $6 \mathrm{~W} / \mathrm{mm}^{2}$ is reached in the right part of the contact patch. An explanation for this result may be that the spin diminishes as a result from the inclination of the wheel rim 

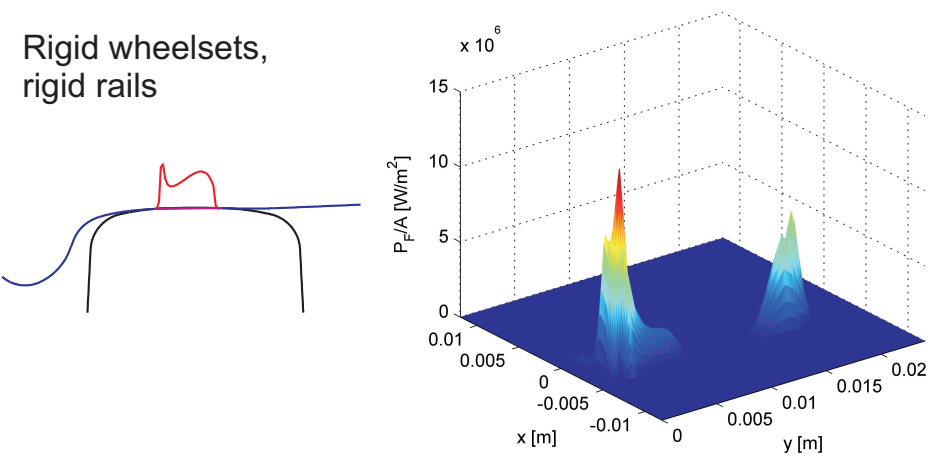

Elastic wheelsets, elastic rails
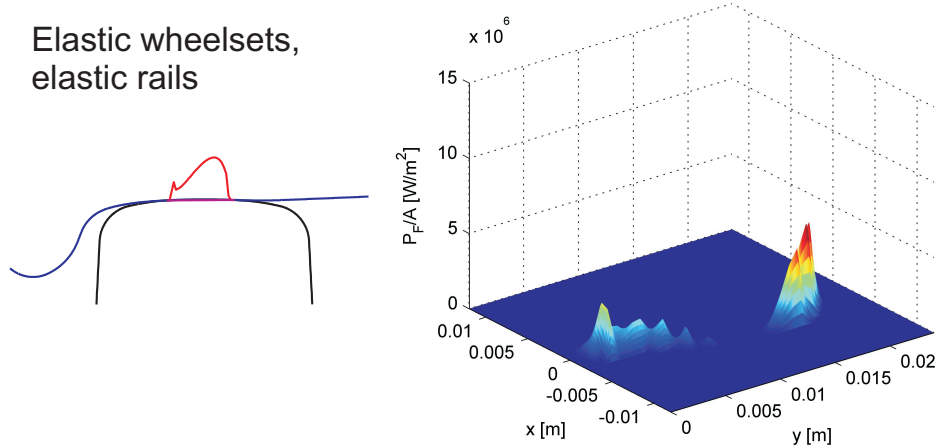

Figure 13: Distribution of the density of the frictional power for $v_{0}=200 \mathrm{~km} / \mathrm{h}$ and $\mu=0.3$.

due to the wheelset's bending.

Of course, it should be kept in mind, that these results are calculated using ideal conditions, i.e. new unworn profiles S1002 and UIC60 and an undisturbed, centred position of the wheelset. In real life, both conditions are hardly fulfilled. However, the result that structural deformations of the wheelset already have an impact on the wheel-rail contact in this "unspectacular" case suggests, that this refined modelling of the vehicle-track system should also be used for the description of more realistic scenarios.

\subsection{Hunting of the wheelset}

The hunting motion is a combined lateral $(y)$ and yaw $(\psi)$ motion of the wheelset, as displayed in Fig. 14. Below a certain driving speed, the so-called critical speed $v_{0, c r i t}$, the hunting motion decreases, so that the wheelset centres itself within the track. If the vehicle is running faster than $v_{0, c r i t}$, a permanent hunting motion can occur. Usually, this is avoided by a proper mechanical design of the vehicle, because in this case high lateral wheel-rail forces can occur, which can cause damage to the track and derailment. 


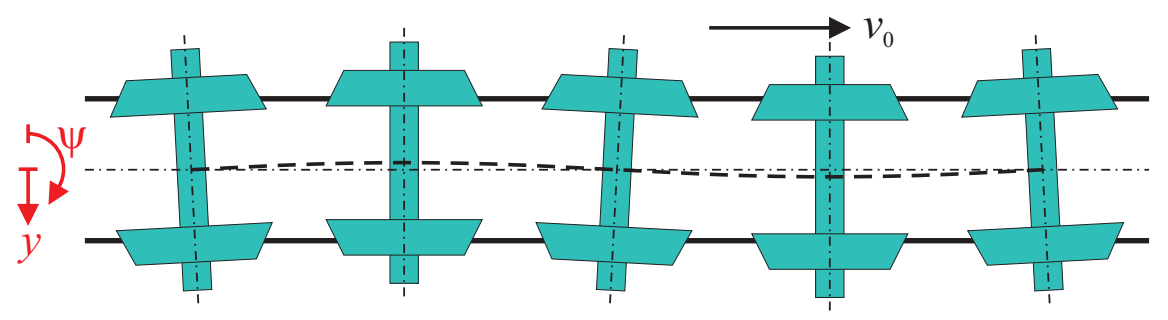

Figure 14: Hunting motion of the wheelset.

Although the scenario of permanent hunting is avoided in regular operation, it is chosen here to study the influence of the structural elasticities on the running behaviour and on the wheel-rail contact. The phase portrait for the lateral motion $y_{W S 1}$ of the first wheelset's centre is displayed in Fig. 15. The curves for the two models

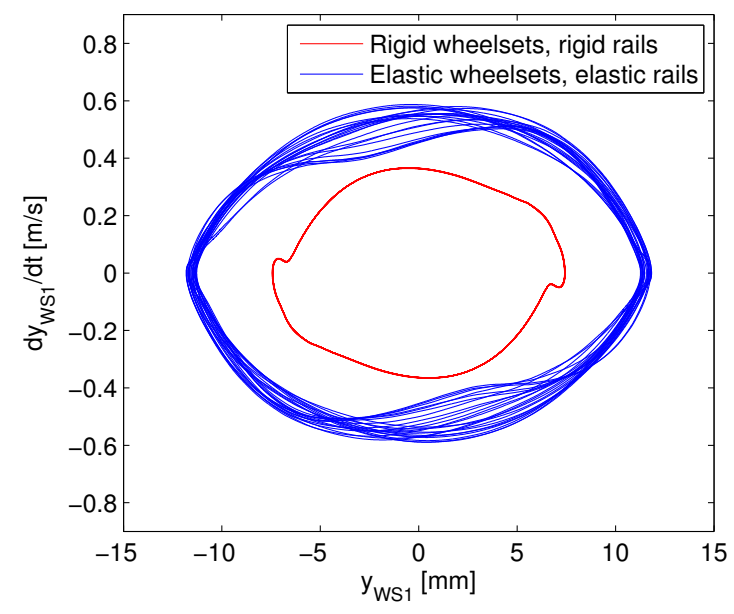

Figure 15: Phase portrait for the lateral motion of the first wheelset's centre at $v_{0}=$ $410 \mathrm{~km} / \mathrm{h}$.

show two differences:

- The lateral amplitudes are larger for the model using elastic wheelsets and elastic rails. Due to the lateral forces, the rail is shifted and the wheelset is deformed, which leads to a larger lateral displacement of the centre.

- The curve for the rigid wheelset on the rigid rails shows sharp bends at $y_{W S 1} \approx$ $6.5 \mathrm{~mm}$ and $y_{W S 1} \approx-6.5 \mathrm{~mm}$, while the curve for the elastic wheelset on elastic rails is smoother. The sharp bends result from the wheel's flange hitting the rail head. If the structural elasticity of the wheelset and the rails is taken into account, the wheelset and the rails act as springs, which are softer than the comparatively stiff wheel-rail contact. Thereby, the impact of the wheel flange and the railhead is cushioned. 
The influence of the structural deformations of the wheelset and the rails on the right wheel-rail contact is displayed in Fig. 16. Since the lateral displacement of the

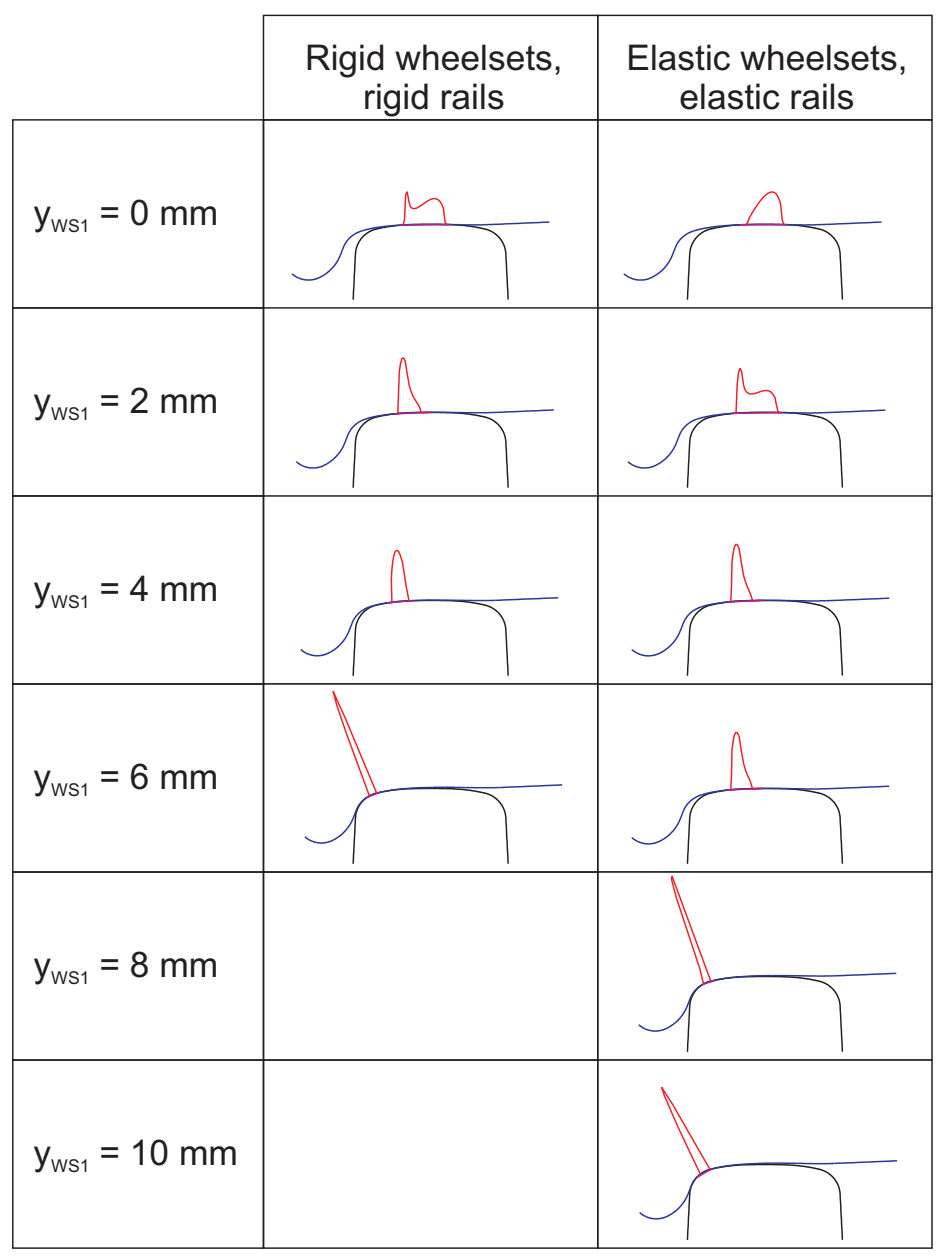

Figure 16: Maximum normal pressure at the right wheel depending on the lateral shift $y_{W S 1}$ of the wheelset.

wheelset doesn't exceeds the range of $-7.5 \mathrm{~mm}<y_{W S 1}<7.5 \mathrm{~mm}$ for rigid wheelsets and rigid rails, no diagrams are available for $y_{W S 1}=8 \mathrm{~mm}$ and $y_{W S 1}=10 \mathrm{~mm}$.

As already observed for the case of centred running, the distribution of the pressure in the contact area is distinctly changed. It can also be seen, that the contact between the flange of the wheel and the gauge corner of the rail occurs at higher lateral displacements of the wheelset, if the structural elasticities are taken into account. This is probably caused by the vertical forces, which were relevant in the case of centred running, but also by the lateral forces. These lateral forces, which guide the wheelset in the case of hunting, provoke a bending of the wheelset and a lateral shifting of the rail. 


\section{Conclusion}

The comparison of the results obtained with two vehicle-track models shows that the structural dynamics of the wheelsets and the rails have in fact an impact on the stress distribution in the wheel-rail contact. Already in the case of centred, undisturbed running of the wheelset, the shape of the contact patch and the distribution of the stresses are changed considerably, which can be explained by the bending of the wheelset. The distribution of the density of the frictional power, which is important for the wear, is also affected distinctly. In the case of hunting, the structural dynamics have a noticeable impact on the running behaviour of the wheelset. This also leads to distinct changes of the stress distribution in the wheel-rail contact. Although the two scenarios, which are investigated here, may be idealised, the refined modelling including the elastic wheelsets and rails should also be used for simulating more realistic cases, especially the running on a disturbed track. This might be the base for a more detailed knowledge of the wear occurring in railway systems, which has a strong impact on their maintenance efforts and thereby on their economics.

\section{References}

[1] J. Piotrowski and H. Chollet, "Wheel-rail contact models for vehicle system dynamics including multi-point contact", Vehicle System Dynamics Vol. 43, No. 67, pp. 455483, June-July 2005.

[2] J.J. Kalker, "Three-dimensional elastic bodies in rolling contact", Kluwer Academic Publishers, Dordrecht, 1990.

[3] P. Diepen, "Horizontaldynamik von Drehgestellfahrzeugen - Berechnung und Optimierung der Laufverhaltens von schnellfahrenden Reisezugwagen mit konventionellen Laufwerken", doctoral thesis, TU Braunschweig, 1991.

[4] K.-H. Kim, "Verschleissgesetz des Rad-Schiene-Systems", doctoral thesis, RWTH Aachen, 1996.

[5] H. Bremer, "Elastic multibody dynamics: A direct Ritz approach", SpringerVerlag, Dordrecht, 2008.

[6] L.D. Landau and E.M. Lifshitz, "Theory of elasticity", Butterworth-Heinemann, Oxford, 1986.

[7] B. Ripke, "Hochfrequente Gleismodellierung und Simulation der FahrzeugGleis-Dynamik unter Verwendung einer nichtlinearen Kontaktmechanik", VDIVerlag, Düsseldorf, 1995.

[8] E.A.H. Vollebregt, "A Gauss-Seidel type solver for special convex programs, with application to frictional contact mechanics", Journal of Optimization Theory and Applications: Vol. 87, No.1, pp. 47-67, October 1995. 